\title{
Nitrofurantoin associated pulmonary fibrosis
}

\section{Olayinka A. Ogundipe*}

Department of Medicine of the Elderly, Royal Infirmary of Edinburgh, 51 Little France Crescent, Edinburgh, Scotland

Received: 27 March 2020

Revised: 29 April 2020

Accepted: 30 April 2020

\section{*Correspondence:}

Dr. Olayinka A. Ogundipe,

Email: ola_ayodele@hotmail.com

Copyright: ( $)$ the author(s), publisher and licensee Medip Academy. This is an open-access article distributed under the terms of the Creative Commons Attribution Non-Commercial License, which permits unrestricted non-commercial use, distribution, and reproduction in any medium, provided the original work is properly cited.

\begin{abstract}
This report describes the case of an 83 years old woman presenting to hospital due to a fall, and acute confusion. On admission, she was diagnosed as having clinical and plain X-ray features of a pneumonia. Following treatment and recovery from the pneumonia, she was noted to have persistent clinical signs of bilateral lung crackles on auscultation. A high-resolution computerised tomography scan showed signs of interstitial lung disease consistent with pulmonary fibrosis. Of relevance was a medication history that indicated oral nitrofurantoin had been initiated in primary care for the long-term prophylaxis of recurrent urinary tract infections. Given the prolonged duration of use of ten years, we considered the possibility of this being an adverse drug reaction in the form of nitrofurantoin associated pulmonary fibrosis. This report also briefly reviews the relevant literature on nitrofurantoin associated pulmonary fibrosis and to promote objectivity in the pharmacovigilance related reporting. The report has been reviewed with the aid of two validated causality assessment systems.
\end{abstract}

Keywords: Adverse drug reaction, Causality, Interstitial lung diseases, Nitrofurantoin, Pharmacovigilance, Pulmonary fibrosis

\section{INTRODUCTION}

Nitrofurantoin is an antibacterial agent that is often prescribed for the treatment of cystitis and uncomplicated urinary tract infections (UTIs). ${ }^{1}$ As UTIs are a common occurrence in older patients, some clinicians also prescribe it prophylactically in an attempt to reduce the incidence of recurrent UTIs. ${ }^{1}$

This report describes an older female patient who developed radiologically confirmed pulmonary fibrosis in the context of long-term use of oral nitrofurantoin.

\section{CASE REPORT}

An 83 year old Caucasian woman was admitted to hospital following a fall with a long lie, and in a confused state.
On admission, the limited available medical history noted secondary progressive multiple sclerosis, recurrent UTIs, hypothyroidism and osteoporosis. Her regular medications were alendronic acid $70 \mathrm{mg}$ once weekly, adcal D3 one tablet twice daily, levothyroxine 100 micrograms once daily, and nitrofurantoin $50 \mathrm{mg}$ every night.

Her observations on admission showed a blood pressure of $157 / 102 \mathrm{mmHg}$, pulse 120 beats per min, respiratory rate 20 breaths per minute, and resting oxygen saturations of $92 \%$ when breathing air. She had multiple soft tissue and skin related injuries from the fall. She was mildly pyrexial, acutely breathless and had bibasal inspiratory crackles. She also showed signs of a fluctuant hypoactive delirium. 
Initial blood tests included: urea $22.5 \mathrm{mmol} / \mathrm{l}$ (2.5-6.6), creatinine $85 \mu \mathrm{mol} / \mathrm{l}$ (60-120), sodium $151 \mathrm{mmol} / \mathrm{l}$ (135145), potassium $2.9 \mathrm{mmol} / \mathrm{l} \quad(3.6-5.0)$, estimated glomerular filtration rate (eGFR) $55 \mathrm{ml} / \mathrm{min} \quad(>60$ $\mathrm{ml} / \mathrm{min} / 1.73 \mathrm{~m}^{2}$ ), and creatine kinase $(\mathrm{CK})$ of $935 \mathrm{U} / \mathrm{l}$ (30-135). Other blood tests revealed that her initial full blood count, serum folate and B12 levels, calcium, thyroid and liver function tests were all within normal ranges.

From a microbiological perspective, urine cultures showed no significant growth. Urinary antigen testing for Legionella pneumophila serogrp1 antigen was negative. In addition, a throat swab for an extensive virology panel was negative (e.g. Coronavirus, Adenovirus, Bocavirus, Influenza $\mathrm{A}$ and $\mathrm{B}$, Metapneumovirus, Mycoplasma pneumonia, Parainfluenza virus types 1-4, Rhinovirus, and Respiratory syncytial virus).

A resting 12 lead electrocardiogram (ECG) was unremarkable. The initial plain chest radiograph confirmed predominant right middle and lower zone consolidation suggestive of a pneumonia (Figure 1).

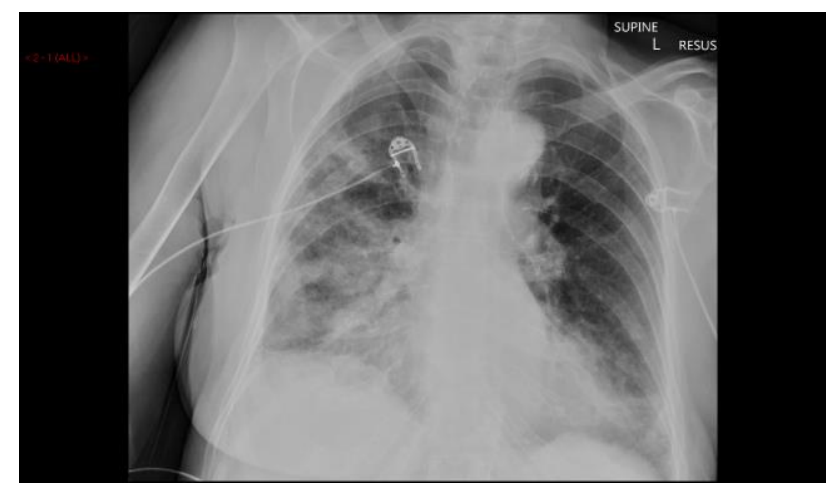

Figure 1: Chest X-ray showing predominant right middle and lower zone consolidation, and also some left basal consolidation (before antibiotic treatment).

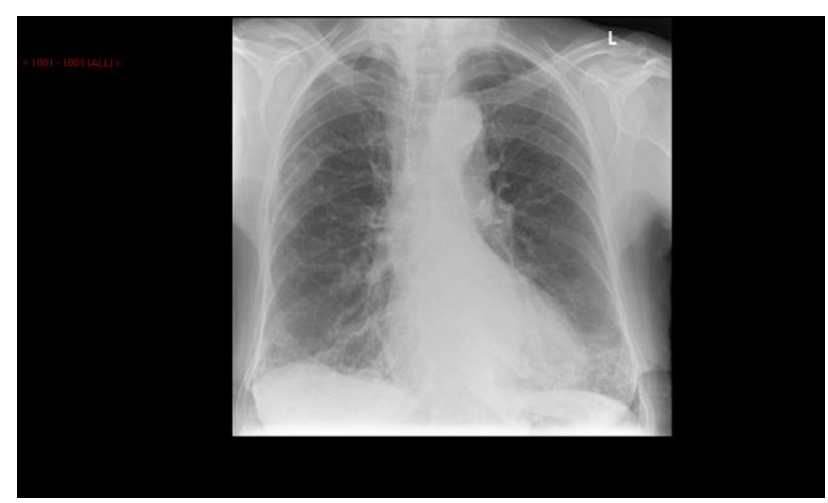

Figure 2: Chest X-ray showing improvement to right middle and lower zone consolidation, and also to the left basal consolidation (after antibiotic treatment).

The working diagnoses included a fall with a long lie, hypoactive delirium, right-side predominant aspiration pneumonia, pre-renal acute kidney injury (AKI) and deranged electrolytes (hypernatraemia and hypokalaemia).

She was admitted directly from the emergency department (ED) to a high dependency unit (HDU) for initial treatment. Her ongoing care was subsequently stepped down to an acute medicine of the elderly (MoE) ward. The patient responded well (both clinically and radiologically) to treatment of the pneumonia (Figure 2).

The specific treatment modalities included a period of oxygen supplementation, and a seven-day course of (intravenous and then oral) amoxicillin and metronidazole, during which her delirium also resolved fully. The pre-renal AKI, elevated CK level and electrolyte abnormalities all progressively normalised with intravenous fluids and relevant electrolyte supplementation. Prophylaxis for venous thromboembolism was initially with subcutaneous unfractionated heparin (during the period of $\mathrm{AKI}$ ), and later with subcutaneous low molecular heparin (after resolution of the AKI). Appropriate pressure area precautions were applied to preserve skin integrity and to promote soft tissue healing over time.

On the acute MoE ward, she was noted to have persistent bilateral lung crackles to the lower and midzones, although now no longer presenting as acutely ill. A clinical suspicion was raised that there may be an alternate (chronic) cause for the non-resolved and lingering respiratory signs, to which she appeared to have become adapted or acclimatised to.

As the delirium had now resolved, the clinical team were able to revisit her history in more depth. It was established that from the perspective of an occupational history, she was a retired administrative and secretarial worker. There was no previous exposure to asbestos nor dust-laden working environments. It was also noted that her family history was unremarkable. Her social history was also retrospectively explored and suggested no history of alcohol excess nor nicotine use. She kept no pets. Her travel history was equally unremarkable. From a functional point of view and prior to the admission, she was fully independent with her activities of daily living. Her medication history was also revisited more closely. There was no regular over the counter drug use and she had never taken illicit drugs. However, she confirmed that the oral nitrofurantoin had been prescribed by her primary care physician with a view to reduce occurrences of her tendency to UTIs. She had been taking the nitrofurantoin continuously for the ten (10) years preceding this acute hospital admission.

Based on the prolonged period of use (ten years) of nitrofurantoin, we entertained the distinct possibility that this might be a delayed presentation of an adverse drug reaction (ADR) i.e., in the form of nitrofurantoin associated pulmonary fibrosis. 
An updated general examination showed no finger clubbing nor peripheral lymphadenopathy. She had no mucocutaneous lesions. There was no reported myalgia nor arthralgia. Her weight was $61.3 \mathrm{~kg}$, height 1.63 metres, and her derived body mass index (BMI) was 23.1.

At this point, further detailed investigations were undertaken to try and establish whether or not this might indeed be a case of nitrofurantoin associated pulmonary fibrosis, or if additional tests would point towards a plausible alternative aetiology.

A retrospective review of a series of full blood count results confirmed that these were in the normal ranges, and in particular showed no indication of eosinophilia.

Immunology and connective tissue disease related tests returned a (non-specifically) positive antinuclear antibody (ANA) screen with a titre of $1 / 640$ homogeneous, but follow-on testing for dsDNA antibodies was negative. Complement C3 and C4 levels were normal, as was her cyclic citrullinated peptide (CCP) level. A detailed extractable nuclear antigen (ENA) panel returned negative for Ro (Sjogren's syndrome: SS-A); La (Sjogren's syndrome: SS-B); SmDP; ribonucleoprotein: RNP70; anti-topoisomerase I or Scl-70S; and Jo-1 antibodies. Myeloperoxidase (MPO) and Proteinase 3 (PR3) related antineutrophil cytoplasmic antibodies (ANCA) were also negative. Anti-histone antibodies were normal at $10 \mathrm{U} / \mathrm{ml}(0.0-40)$. Aspergillus precipitins $(\mathrm{IgG})$ fungal antibody levels were normal at $17.4 \mathrm{mg} \mathrm{A} / \mathrm{l}(0-58)$.

A high-resolution non-contrast computerised tomography (HRCT) lung scan (Figures 3-5) demonstrated evidence of mild-moderate pulmonary fibrosis with septal thickening, scattered ground-glass opacity and honeycombing, with a mostly peripheral distribution.

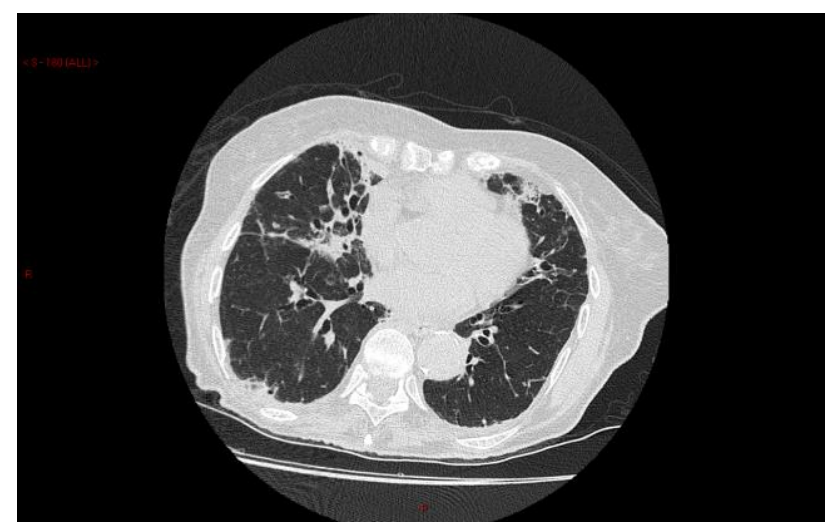

Figure 3: Section of HRCT scan showing background changes of pulmonary fibrosis.

Fibrotic changes were noted to be most marked in the lower zones. Traction bronchiectasis was present, mostly demonstrated in the middle lobe and lingula. Additional patchy airspace infiltrates were noted in the right upper and middle lobes and lingula which were likely part of the fibrotic process, but superadded infection was raised as a possibility. There was no pleural effusion but a shallow pericardial effusion was noted. Marked coronary artery calcification was noted. There were no size significant thoracic lymph nodes. There were old bilateral rib fractures but no destructive bone lesion. The summary opinion was: CT confirms pulmonary fibrosis with honeycombing and traction bronchiectasis. Overall appearances would be consistent with nitrofurantoin associated fibrosis.

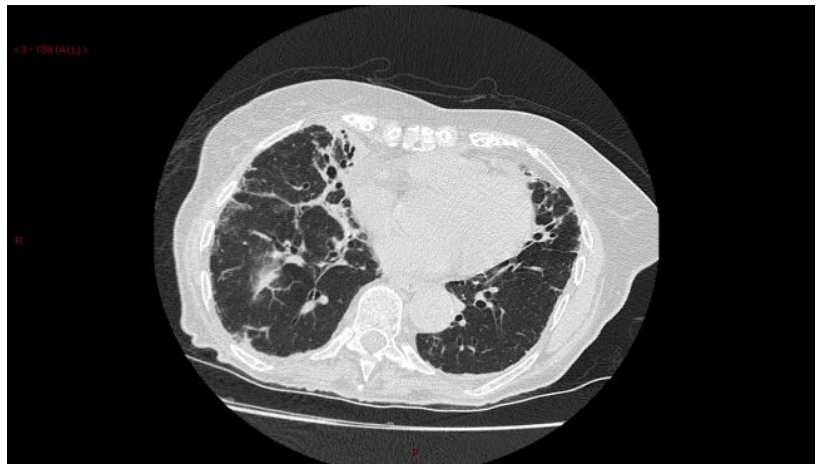

Figure 4: Section of HRCT scan showing further changes of pulmonary fibrosis with septal thickening.



Figure 5: Section of HRCT scan showing changes of pulmonary fibrosis with ground-glass opacity and honeycombing.

The further detailed evaluations described above led us to conclude that this was most likely a nitrofurantoin associated pulmonary fibrosis i.e. rather than being due to an alternative cause or being idiopathic in nature. The justification for this inference was the context of noting persistent respiratory symptoms and signs, being duly informed by the relevant medication history, and the supportive findings on advanced radiology images.

The oral nitrofurantoin therapy was discontinued. Although the directly observed inpatient timelines were short ( four weeks), the initial response noted to withdrawal of the nitrofurantoin was encouraging. She was successfully weaned off oxygen, and attained resting oxygen saturations of $97 \%$ when breathing air. 
She subsequently completed a period of comprehensive geriatric assessment and rehabilitation to improve her mobility and exercise tolerance, and successfully achieved a supported discharge back home. On discharge from hospital, the patient's ongoing care was referred back to her general practitioner. Following discharge, further follow-up and support was coordinated via our tertiary respiratory service which offers access to a specialist pulmonary fibrosis clinic.

\section{DISCUSSION}

Nitrofurantoin is a nitrofuran derivative and an antibacterial agent. It is often used in treating cystitis and uncomplicated UTIs. ${ }^{1}$ It is also prescribed prophylactically to reduce the incidence of recurrent UTIs. ${ }^{1}$

From an epidemiological perspective, UTIs are common in older patients and also occur at a higher prevalence in women. Women of childbearing age and pregnant women with UTIs may also be prescribed nitrofurantoin, although its use is discouraged in the last trimester to reduce the risks of haemolytic jaundice in the newborn. ${ }^{1}$

In the United Kingdom (UK), although nitrofurantoin is licenced for both treatment and prophylaxis of UTIs, there have been additional arguments against the use of nitrofurantoin as a long-term prophylactic strategy against UTIs. In the latter context, a commonly cited concern is the potential risk of antibiotic resistance.

\section{Some basic pharmacodynamic and pharmacokinetic considerations}

Nitrofurantoin is available in an oral formulation (e.g., as specially formulated macrocrystalline-based tablets or capsules). ${ }^{1}$ The oral capsule offers an advantage of a modified release preparation, the latter of which might lessen the impact of gastrointestinal side-effects. An oral suspension is also available although very expensive. Dependent upon the specific formulation used, nitrofurantoin is generally described as possessing fair to good bioavailability. The drug undergoes predominant hepatic metabolism. Its excretion is mainly renal, and to a lesser extent biliary.

The general recommendation is to avoid nitrofurantoin when a patient's eGFR is less than $45 \mathrm{ml} /$ minute $/ 1.73 \mathrm{~m}^{2}{ }^{1}$ However, there is additional guidance for when nitrofurantoin may be used (with caution) in patients with eGFR of between 30 and $44 \mathrm{ml} /$ minute $/ 1.73 \mathrm{~m}^{2}{ }^{1}$ In the latter scenario, the clinician is expected to weigh up the perceived benefits versus risks. If available, clinicians should also consider other accessible information (e.g., microbiological sensitivities). Where possible, clinicians should concurrently aim to limit the duration of treatment to short courses (e.g., 3 to 7 days; although this may be dependent upon the specific indication). ${ }^{1}$
As a general note, nitrofurantoin is used extensively, is mostly well tolerated and has an overall reasonable sideeffect profile. ${ }^{1}$ That said, it has some more commonly recognised gastrointestinal side-effects such as nausea, vomiting and diarrhoea. ${ }^{1}$

However, clinicians also need to be increasingly aware of the potential for other (rarer) ADRs with short-term, longer-term or prolonged use of nitrofurantoin. ${ }^{1-4}$ In particular, clinicians need greater awareness of possible pulmonary ADRs of nitrofurantoin (e.g., pulmonary hypersensitivity, pulmonary fibrosis, other pulmonary reactions including lupus-like syndromic presentations, etc); neurological reactions (e.g., peripheral neuropathies, idiopathic intracranial hypertension, etc); blood dyscrasias (e.g., eosinophilia, granulocytopenia, leucopenia, haemolytic anaemia, aplastic anaemia, thrombocytopenia); and the risks of organ dysfunction (e.g., hepatitis, pancreatitis). Other rare ADRs include cutaneous manifestations such as DRESS (drug reaction with eosinophilia and systemic symptoms) syndrome and the Stevens-Johnson syndrome. .,3 $^{1,3}$

\section{The index cases}

Further history established that the index case had been prescribed daily oral nitrofurantoin from primary care for the prevention of recurrent UTIs, and that she had been taking the medication continuously for ten years preceding this acute hospital admission. The duration of the prolonged prescription was validated via direct telephone contact with her primary care team. The patient offered what we judged to be reliable self-attestation to her concordance with use of the medication over this period. Based on the clinical findings and supportive radiological evidence, we concluded that this older female patient had developed pulmonary fibrosis in association with prolonged use of oral nitrofurantoin. There was no suggestion of other symptoms or signs of additional significant adverse effects of the prolonged nitrofurantoin therapy e.g., peripheral neuropathy, blood dyscrasias, drug induced hepatitis, renal disease, or cutaneous manifestations.

An acknowledged limitation is that the diagnosis in this case was entertained on clinical grounds, albeit with corroborating evidence arising from the supportive radiological findings, rather than through a formal lung biopsy. Therefore, and although unlikely, it is theoretically still plausible that the noted pulmonary fibrosis was in fact 'idiopathic,' rather than truly medication-associated. In addition, we duly considered the known, albeit rare association between nitrofurantoin use and pulmonary fibrosis. ${ }^{1,2}$ Consequently, in the index case a diagnostic or confirmatory lung biopsy was not deemed to be clinically justified nor necessitated. The justification for this decision took cognisance of the protracted duration of therapy, and the fact that characteristic and advanced findings of pulmonary fibrosis were noted on the patient's HRCT. 
Table 1: Naranjo ADRS algorithm. ${ }^{5}$

Questionnaire applied to index case report

Are there previous conclusive reports on this reaction?

Yes (+1) No (0) Do not know or not done (0)

Did the adverse event appear after the suspected drug was given?

Yes (+2) No (-1) Do not know or not done (0)

Did the adverse reaction improve when the drug was discontinued or a specific antagonist was given?

Yes (+1) No (0) Do not know or not done (0)

Did the adverse reaction appear when the drug was re-administered?

Yes (+2) No (-2) Do not know or not done (0)

Are there alternative causes that could have caused the reaction?

Yes (-1) No (+2) Do not know or not done (0)

Did the reaction reappear when a placebo was given?

Yes (-1) No (+1) Do not know or not done (0)

Was the drug detected in any body fluid in toxic concentrations?

Yes (+1) No (0) Do not know or not done (0)

Was the reaction more severe when the dose was increased or less severe when the dose was decreased?

Yes (+1) No (0) Do not know or not done (0)

Did the patient have a similar reaction to the same or similar drugs in any previous exposure?

Yes (+1) No (0) Do not know or not done (0)

Was the adverse event confirmed by any objective evidence?

Yes (+1) No (0) Do not know or not done (0)

$>9=$ definite ADR

$5-8=$ probable ADR

1-4 = possible ADR

$0=$ doubtful ADR

A derived total score of 3 translates to a classification of a 'possible' adverse drug reaction (ADR).

Table 2: WHO-UMC causality categories. ${ }^{8}$

\begin{tabular}{|c|c|}
\hline Causality term & Causality term assessment criteria* \\
\hline \multirow{5}{*}{ Certain } & Event or laboratory test abnormality, with plausible time relationship to drug intake \\
\hline & Cannot be explained by disease or other drugs \\
\hline & Response to withdrawal plausible (pharmacologically, pathologically) \\
\hline & $\begin{array}{l}\text { Event definitive pharmacologically or phenomenologically (i.e. an objective and specific } \\
\text { medical disorder or a recognised pharmacological phenomenon) }\end{array}$ \\
\hline & Rechallenge satisfactory, if necessary \\
\hline \multirow{4}{*}{ Probable/likely } & Event or laboratory test abnormality, with reasonable time relationship to drug intake \\
\hline & Unlikely to be attributed to disease or other drugs \\
\hline & Response to withdrawal clinically reasonable \\
\hline & Re-challenge not required \\
\hline \multirow{5}{*}{$\begin{array}{l}\text { Possible/ } \\
\text { unlikely }\end{array}$} & Event or laboratory test abnormality, with reasonable time relationship to drug intake \\
\hline & Could also be explained by disease or other drugs \\
\hline & Information on drug withdrawal may be lacking or unclear \\
\hline & $\begin{array}{l}\text { Event or laboratory test abnormality, with a time to drug intake that makes a relationship } \\
\text { improbable (but not impossible) }\end{array}$ \\
\hline & Disease or other drugs provide plausible explanations \\
\hline \multirow{2}{*}{$\begin{array}{l}\text { Conditional/ } \\
\text { unclassified }\end{array}$} & Event or laboratory test abnormality \\
\hline & More data for proper assessment needed, or additional data under examination \\
\hline \multirow{3}{*}{$\begin{array}{l}\text { Unassessable/ } \\
\text { unclassifiable }\end{array}$} & Report suggesting an adverse reaction \\
\hline & Cannot be judged because information is insufficient or contradictory \\
\hline & Data cannot be supplemented or verified \\
\hline
\end{tabular}

Examples of these detailed radiological findings included the presence of a significant degree of lung scarring, septal thickening and traction bronchiectasis. The HRCT also demonstrated 'ground glass' and 'honeycomb' changes occurring in typical areas and distribution, such as in the patient's lung bases and lung peripheries. In this 
context, we also judged it clinically inappropriate to continue the medication or to consider a 'medication rechallenge' by re-introduction of the nitrofurantoin after a period of discontinuation.

It has been previously reported in the medical literature that the timely discontinuation of nitrofurantoin therapy reportedly results in significant resolution of symptoms in many cases. ${ }^{2}$ However, we postulate that it is unlikely that the established structural changes of pulmonary fibrosis noted on this patient's HRCT scan would resolve fully. Nevertheless, the belated withdrawal of the nitrofurantoin might forestall further clinical deterioration in this patient. As the patient also has secondary progressive multiple sclerosis, cessation of the long-term nitrofurantoin could possibly reduce the risk of her developing other rare but recognised side-effects of nitrofurantoin such as peripheral neuropathy, the latter of which could have a further adverse functional impact on her quality of life.

\section{Application of ADR causality assessment systems to the index case}

The use of previously validated causality assessment systems (CAS) can be used to improve the process of ADR-related case reporting. This has the potential to promote and enhance objectivity in reporting when medications are suspected of, or reported as being associated with ADRs. ${ }^{5-7}$ These CAS are not perfect, but may nevertheless support and potentially augment pharmacovigilance related assessments. Adoption of CAS may also allow for more structured and transparent ADR reporting of case reports or case series. For example, even with conservative scoring of this case report, applying the Naranjo adverse drug reaction probability scale (Table 1) translates into a score of 3 , which equates to a 'possible' ADR classification. ${ }^{5}$ Equally, a 'possible' ADR classification for this case report is obtained by the stringent application of another validated CAS, i.e. the WHO-UMC method (Table 2). ${ }^{8}$

\section{CONCLUSION}

This report describes an older female patient with clinical and radiological evidence suggestive of pulmonary fibrosis associated with the prolonged use of nitrofurantoin. The case illustrates the importance of periodically reviewing the clinical history and drug history for possible ADRs. These may reveal associations linked to the use of a medications, the duration of use, the dosing regimen, and/or the pattern of ADRs that may be described or noted by patients, carers/families, or healthcare-providers.

Furthermore, the report serves as an important clinical reminder that may prove relevant to a range of clinicians e.g., primary care or hospital-based physicians, and also clinicians with extended roles e.g., pharmacy prescribers, nurse prescribers, etc. Clinicians and prescribers should familiarise themselves with ADR reporting systems or schemes for the individual countries in which they practice. ${ }^{9}$ They should also routinely engage in ADR reporting as this promotes the principles of pharmacovigilance.

\section{Authorship statement}

OAO conceived the idea for the article, prepared the initial draft, undertook the critical review and edited the manuscript's final version.

\section{Funding: No funding sources \\ Conflict of interest: None declared \\ Ethical approval: Not required}

\section{REFERENCES}

1. British National Formulary. National Institute for Health and Care Excellence. (c) NICE 2019. Available at: https://bnf.nice.org.uk/drug/nitrofuran toin.html. Accessed on 29 April 2020.

2. Syed H, Bachuwa G, Upadhaya S, Abed F. Nitrofurantoin-induced interstitial pneumonitis: albeit rare, should not be missed. BMJ Case Rep. 2016;10:1136.

3. Leao RN, Barreto P, Leao RR, Ribeiro JV. Nitrofurantoin: cause of DRESS syndrome. BMJ Case Rep; 2013.

4. Agarwal A, Agrawal A, Nathan K, Roy S. Rare adverse effect of a common drug: nitrofurantoininduced ANCA-associated vasculitis. BMJ Case Rep; 2015.

5. Naranjo CA, Busto U, Sellers EM. A method for estimating the probability of adverse drug reactions. Clin Pharmacol Ther. 1981;30(2):239-45.

6. Busto U, Naranjo CA, Sellers EM. Comparison of two recently published algorithms to assess the probability of adverse drug reactions. $\mathrm{Br} \mathrm{J}$ Clin Pharmacol. 1982;13(2):223-7.

7. Kramer MS, Leventhal JM, Hutchinson TA, Feinstein AR. An algorithm for the operational assessment of adverse drug reactions-I. Background, description, and instructions for use. JAMA. 1979;242(7):623-32.

8. The use of the WHO-UMC system for standardized case causality assessment. Uppsala Monitoring Centre. Available at: https://www.who.int/medic ines/areas/quality_safety/safety_efficacy/WHO causality_assessment.pdf. Accessed on 29 April 2020.

9. Yellow Card. (C) Crown Copyright 2019. Available at: https://yellowcard.mhra.gov.uk. Accessed on 29 April 2020.

Cite this article as: Ogundipe OA. Nitrofurantoin associated pulmonary fibrosis. Int J Basic Clin Pharmacol 2020;9:985-90. 